\title{
Finite-time Tracking Control for Nonholonomic Wheeled Mobile Robot Using Adaptive Fast Nonsingular Terminal Sliding Mode
}

Hao Xie ( $\nabla$ hxie@swin.edu.au )

Swinburne University of Technology - Hawthorn Campus: Swinburne University of Technology https://orcid.org/0000-0002-6825-8433

Jinchuan Zheng

Swinburne University of Technology - Hawthorn Campus: Swinburne University of Technology Zhe Sun

Zhejiang University of Technology

Rifai Chai

Swinburne University of Technology - Hawthorn Campus: Swinburne University of Technology Hai Wang

Murdoch University

\section{Research Article}

Keywords: Trajectory tracking, Mobile robot, Finite-time control, Sliding mode control

Posted Date: March 7th, 2022

DOI: https://doi.org/10.21203/rs.3.rs-1304206/v1

License: (c) (1) This work is licensed under a Creative Commons Attribution 4.0 International License. Read Full License 


\title{
Finite-time tracking control for nonholonomic wheeled mobile robot using adaptive fast nonsingular terminal sliding mode
}

\author{
Hao Xie ${ }^{1 *}$, Jinchuan Zheng ${ }^{1}$, Zhe Sun ${ }^{2}$, Hai Wang ${ }^{3}$ and Rifai \\ $\mathrm{Chai}^{1 \dagger}$
}

${ }^{1 *}$ School of Science, Computing and Engineering Technologies, Swinburne University of Technology, Melbourne, 3122, Victoria, Australia.

${ }^{2}$ Department of Automation, Zhejiang University of Technology, Hangzhou, 310023, Zhejiang, China.

${ }^{3}$ Discipline of Engineering and Energy, Centre of Water, Energy, and Waste, Murdoch University, Perth, 6150, WA, Australia.

\author{
*Corresponding author(s). E-mail(s): hxie@swin.edu.au; \\ Contributing authors: jzheng@swin.edu.au; \\ sunzhe726@zjut.edu.cn; Hai.Wang@murdoch.edu.au; \\ rchai@swin.edu.au; \\ †These authors contributed equally to this work.
}

\begin{abstract}
System uncertainties and external disturbances are the major causes of the trajectory tracking performance degradation in nonholonomic wheeled mobile robots (NWMRs). In this article, an adaptive fast nonsingular terminal sliding mode dynamic control (AFNTSMDC) method is proposed to provide enhanced robust and finite-time tracking performance for the NWMR. The proposed AFNTSMDC is a systematic design method based upon both the kinematic and dynamic model of the NWMR. The AFNTSMDC controller has a simple form without singularity issue in the control input, which makes it practically implementable. The finite-time stability of the proposed tracking-error function is also proved using the Lyapunov function. Finally, circular trajectory tracking experiments are conducted to validate the robustness and convergence rate of the proposed
\end{abstract}


AFNTSMDC scheme in comparison with the existing methods including classic kinematic control (CKC), robust sliding mode kinematic control (RSMKC), and conventional sliding mode dynamic control (CSMDC) in the presence of uncertainties and external disturbances.

Keywords: Trajectory tracking Mobile robot Finite-time control Sliding mode control

\section{Introduction}

Trajectory tracking control of nonholonomic wheeled mobile robots (NWMRs) has attracted much attention in past decades due to their wide use in various applications [1]. In addition, they pose a challenge to control practitioners since the mechanism of NWMRs is characterized by nonholonomic constraints and the inherent nonlinearity limits the effectiveness of linear controllers [2].

Achieving a more robust and faster trajectory tracking performance is a challenging task due to the system nonlinearities, model uncertainties and disturbances, and many efforts have been devoted to this research field [3-7]. Fast response and strong robustness against system uncertainties and disturbances are crucial objectives in tracking control tasks which are also the key features of sliding mode control (SMC) techniques $[8,9]$. Although many studies on applying SMC techniques for tracking control of the NWMR have been published, it is worth paying attention to the question of how to utilize more advanced SMC to achieve better control performance. The work in [10] proposed an SMC law for trajectory tracking of a NWMR by means of computed torque technique and representing the posture in polar coordinate, which is subjected to constraints on the heading angle and desired velocities. This was further extended in [11], which loosened the constraint of the former study in regard to heading angles and desired velocities for the mobile robot by designing three separate controllers under three operating conditions. However, there is an inherent drawback of the control input singularity around the origin in both aforementioned papers.

Meanwhile, attempts have been made to apply SMC law in Cartesian coordinates [12-18]. Among those studies, cascaded control systems, namely inner-outer loop control structures [13-15] are adopted. The inner loop controller is targeted at velocity following control for the NWMR whilst the outer loop focuses on designing a model-free kinematic controller. According to the posture tracking errors, the outer loop controller generates corresponding velocity commands to the inner loop which guarantees actual velocities to converge to the velocity commands. The capability for the NWMR to achieve the desired performance is built upon the assumption of perfect velocity tracking [19] which, however, may not hold in practice depending on the accuracy of the inner-loop control. In [13], a super-twisting SMC method is developed with a proportional-derivative (PD) controller which increases the robustness 
by mitigating the influence of neglected dynamics, but singularity issue also arises based on the designed sliding surface. With a similar PD controller, the work [14] proposed an adaptive fuzzy SMC method which reduces the system chattering by replacing the adaptive fuzzy logic with the traditional discontinuous portion in SMC. By combining event-triggered structure with a robust SMC, the control input singularity was avoided in [15].

Note that most of the aforementioned papers only guarantee the asymptotic stability of the mobile robot system which means that they may achieve convergence in infinite settling time and a fast convergence rate may not be accomplished. Finite-time tracking control of nonholonomic mobile robots quickly became an emerging topic [20-22]. In [21], a cascaded control system is formed by splitting the error dynamics of the mobile robot into two subsystems. Based on the controller developed, finite-time convergence is guaranteed but only when the velocities and their derivatives are within a limited range. Additional modelling parameters are considered in the work [22] to improve the control accuracy. Likewise, two subsystems are formed with two adaptive sliding mode controllers to strengthen the system robustness. However, the control system only guarantees that the tracking errors can converge to a region instead of zero and the desired angular velocity cannot reach zero, which means that a straight-path following task is not achievable. In addition, both studies [21], [22] only illustrated simulation results and thus the practical effectiveness for their controllers is still questionable.

Motivated by [15], [22], and [23], we proposed an AFNTSMDC method in this paper, whose main contribution is to provide a unified control scheme compared to the classic cascaded control structure. Thus, the assumption of perfect velocity tracking is not needed. This also simplifies the design process as there is no need to design a kinematic controller and a dynamic velocity controller separately. This also leads to a reduction in the number of tuning parameters and tuning processes. In addition, the AFNTSMDC method guarantees finitetime convergence of the tracking error towards zero. It is also more robust against model uncertainties and external disturbances. By properly designing the tracking-error function, an alternative solution to eliminating the control input singularity is also proposed.

The remaining part of the paper is organized as follows. The plant model of the NWMR system consisting of parametric uncertainties and external disturbances is formulated in Section 2. Section 3 describes the AFNTSMDC design method, and the stability analysis and parameters selection are also elaborated. Section 4 presents the experimental performance of the developed controller on the NWMR platform in comparison with other existing methods. The conclusion is drawn in Section 5 .

\section{Problem formulation}

In this section, the dynamics and kinematics of NWMRs are discussed. A schematic top view is shown in Fig. 1, where the mass center of the mobile 


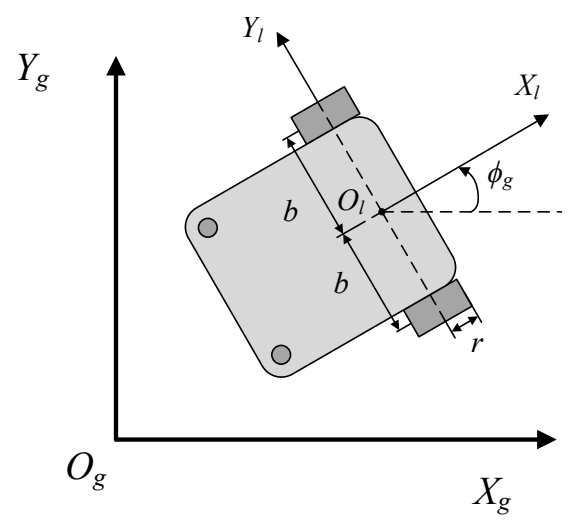

Fig. 1: Top view of a nonholonomic wheeled mobile robot [12].

robot is denoted by $O_{l}$ and it is presumed to be located at the middle of the two front driving wheels. Thus, $\left\{O_{g} X_{g} Y_{g}\right\}$ is the reference coordinate system, whereas $\left\{O_{l} X_{l} Y_{l}\right\}$ is the coordinate system fixed to the mobile robot. The position of the robot is completely specified by the vector $p=\left[\begin{array}{lll}x_{g} & y_{g} & \phi_{g}\end{array}\right]^{T}$. Then, a dynamic model for the NWMR can be described as [22]

$$
\bar{M}(p) \ddot{p}+\bar{F}(\dot{p})+\tau_{d}=\bar{B}(p) \tau-A^{T}(p) \lambda
$$

where $\bar{M}(p) \in R^{r \times r}$ is a positive symmetric definite inertia matrix, $\bar{F}(\dot{p}) \in$ $R^{r \times 1}$ denotes the surface friction, $\tau_{d}$ denotes the lumped bounded unknown disturbance and unmodelled dynamics, $\tau \in R^{n \times 1}$ is the input vector. $A^{T}(p) \in$ $R^{m \times r}$ is the matrix associated with constraints, and $\lambda \in R^{m \times 1}$ is the constraint forces. The matrices $\bar{M}(p), \bar{B}(p), A^{T}(p), \tau, \tau_{d}$ and $\lambda$ are given as follows:

$$
\begin{aligned}
& \bar{M}(p)=\left[\begin{array}{ccc}
m & 0 & 0 \\
0 & m & 0 \\
0 & 0 & I
\end{array}\right], A^{T}(p)=\left[\begin{array}{c}
-\sin \left(\phi_{g}\right) \\
\cos \left(\phi_{g}\right) \\
0
\end{array}\right] \\
& \bar{B}(p)=\frac{1}{r}\left[\begin{array}{cc}
\cos \left(\phi_{g}\right) & \cos \left(\phi_{g}\right) \\
\sin \left(\phi_{g}\right) & \sin \left(\phi_{g}\right) \\
b & -b
\end{array}\right], \tau=\left[\begin{array}{l}
\tau_{1} \\
\tau_{2}
\end{array}\right], \tau_{d}=\left[\begin{array}{c}
\tau_{d 1} \\
\tau_{d 2}
\end{array}\right] \\
& \lambda=-m\left(\dot{x}_{g} \cos \left(\phi_{g}\right)+\dot{y}_{g} \sin \left(\phi_{g}\right) \dot{\phi}_{g}\right)
\end{aligned}
$$

where $m$ is the total mass of the NWMR including load uncertainty, $I$ is the moment of inertia of the mobile robot, $\tau_{i}(i=1,2)$ indicates the torques generated by the right and left wheels, and $\tau_{d i}(i=1,2)$ denotes the external disturbances.

The nonholonomic kinematic constraints are described by

$$
A(p) \dot{p}=0
$$


The kinematic model for the NWMR can be described as

$$
\dot{p}=S(p) z
$$

where

$$
S(p)=\left[\begin{array}{cc}
\cos \left(\phi_{g}\right) & 0 \\
\sin \left(\phi_{g}\right) & 0 \\
0 & 1
\end{array}\right], z=\left[\begin{array}{c}
v \\
\omega
\end{array}\right]
$$

with $v$ and $\omega$ denote the forward velocity and angular velocity, respectively. From (4), it follows that

$$
\ddot{p}=S(p) \dot{z}+\dot{S}(p) z .
$$

Substituting (6) into (1) yields

$$
\bar{M}(p)(S(p) \dot{z}+\dot{S}(p) z)+\bar{F}(\dot{p})+\tau_{d}=\bar{B}(p) \tau-A^{T}(p) \lambda .
$$

Multiplied (7) by $S^{T}(p)$ on the left, the system dynamics (1) can be rewritten as

$$
M \dot{z}=B \tau+d
$$

where

$$
M=\left[\begin{array}{cc}
m & 0 \\
0 & I
\end{array}\right], B=\frac{1}{r}\left[\begin{array}{cc}
1 & 1 \\
b & -b
\end{array}\right], d=\left[\begin{array}{l}
d_{1} \\
d_{2}
\end{array}\right]=-S^{T}(p)\left(\bar{F}(\dot{p})+\tau_{d}\right) .
$$

In addition, the following parametric uncertainties are considered:

$$
M=M_{0}+\Delta M
$$

with

$$
M_{0}=\left[\begin{array}{cc}
m_{0} & 0 \\
0 & I_{0}
\end{array}\right], \Delta M=\left[\begin{array}{cc}
\Delta m & 0 \\
0 & \Delta I
\end{array}\right]
$$

where $m_{0}$ and $I_{0}$ denote the nominal model parameters and $\Delta m$ and $\Delta I$ denotes the corresponding uncertainties, respectively.

Combining (10) and (9), we can obtain the dynamic model for the NWMR as follows:

$$
M_{0} \dot{z}=B \tau-\delta
$$

where $\delta=\Delta M \dot{z}+d$ represents the reformatted uncertainty to system (9), which is assumed to be bounded by

$$
\left|M_{0}^{-1} \delta\right|<c_{0}+c_{1} z
$$




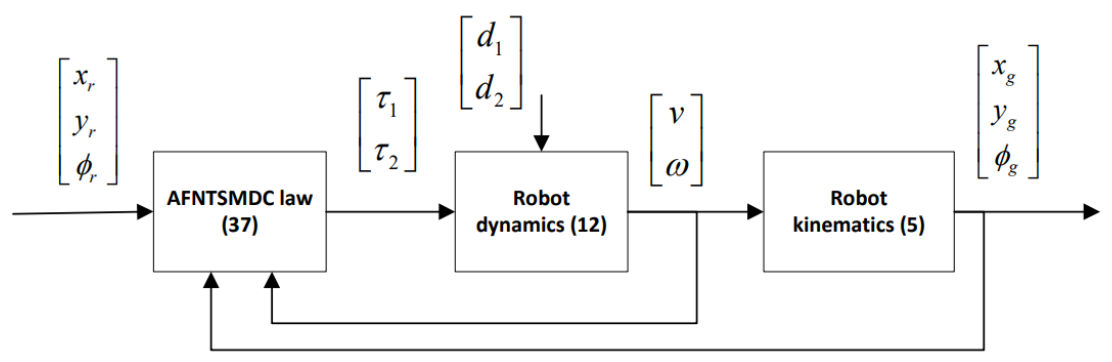

Fig. 2: Block diagram of the proposed unified control structure for NWMR systems.

where $c_{0}$ and $c_{1}$ are unknown but bounded positive numbers. The term $c_{0}$ denotes the upper bound of the external disturbance and surface friction. Meanwhile, $c_{1}$ denotes the upper bound of the dynamic impact caused by modelling imprecision.

In the sequel, the control design for the AFNTSMDC will be constructed according to the kinematic model in (4) and dynamic model in (11) formulated for the NWMR.

\section{Control design}

In this section, the AFNTSMDC design method will be presented such that the NWMR is able to track the desired trajectory command accurately in the presence of system uncertainties and external disturbances in a finite time. To achieve this goal, we will first define a tracking-error function. Then, a fast nonsingular terminal sliding surface with an adaptive reaching law will be developed, based on which the finite-time convergence is achieved, the singularity issue is resolved, and the unified control scheme is developed. Thus, the AFNTSMDC law is constructed with both the velocity and position as feedback signals and the motor torques as the control input.

\subsection{Construction of the AFNTSMDC}

First, define a tracking error vector [19] for the NWMR as

$$
p_{e}=\left[\begin{array}{l}
x_{e} \\
y_{e} \\
\phi_{e}
\end{array}\right]=R(p)\left(p_{r}-p\right)
$$


with

$$
R(p)=\left[\begin{array}{ccc}
\cos \left(\phi_{g}\right) & \sin \left(\phi_{g}\right) & 0 \\
-\sin \left(\phi_{g}\right) & \cos \left(\phi_{g}\right) & 0 \\
0 & 0 & 1
\end{array}\right]
$$

where $p_{r}=\left[\begin{array}{lll}x_{r} & y_{r} & \phi_{r}\end{array}\right]^{T}$ is the desired trajectory posture and its kinematics can be modeled as

$$
\dot{p}_{r}=S\left(p_{r}\right) z_{r}
$$

where $z_{r}=\left[\begin{array}{ll}v_{r} & \omega_{r}\end{array}\right]^{T}$ is the desired velocity, $v_{r}$ denotes the desired forward velocity, and $\omega_{r}$ denotes the desired angular velocity which can be calculated by

$$
\begin{aligned}
v_{r} & =\sqrt{\dot{x}_{r}^{2}+\dot{y}_{r}^{2}} \\
\omega_{r} & =\dot{\phi}_{r}=\frac{\dot{x}_{r} \ddot{y}_{r}-\ddot{x}_{r} \dot{y}_{r}}{\dot{x}_{r}^{2}+\dot{y}_{r}^{2}} .
\end{aligned}
$$

Combining (13) and the kinematic model described in (4), one can obtain the error model for the trajectory tracking control as

$$
\dot{p}_{e}=F+G z
$$

in which

$$
F=\left[\begin{array}{c}
v_{r} \cos \left(\phi_{e}\right) \\
v_{r} \sin \left(\phi_{e}\right) \\
w_{r}
\end{array}\right], G=\left[\begin{array}{cc}
-1 & y_{e} \\
0 & -x_{e} \\
0 & -1
\end{array}\right]
$$

Differentiating (13), we have

$$
\ddot{p}_{e}=\dot{F}+\dot{G} z+G \dot{z} .
$$

Combining (11) with (20), we obtain

$$
\ddot{p}_{e}=\dot{F}+\dot{G} z+G M_{0}^{-1}(B \tau-\delta) .
$$

Second, we shall introduce a new tracking-error function

$$
\xi=\left[\begin{array}{c}
x_{e} \\
\phi_{e}+\frac{\rho}{\left|x_{e}\right|+\rho} \tan ^{-1} y_{e}
\end{array}\right]
$$

where $0<\rho<1$ is the control parameter to be tuned. Note that for $0<\rho<1$ and any $x_{e} \in R$, the expression $\frac{\rho}{\left|x_{e}\right|+\rho} \in(0,1]$, which can be regarded as a weighting parameter to adjust the convergence rate of $y_{e}$ and $\phi_{e}$. It will be 
shown later that once $\xi$ converges to zero, the tracking error vector $p_{e}$ will converge to zero accordingly. Hence, our control objective is now converted to enabling the finite-time convergence of $\xi$ through the proposed method.

Taking the first-order derivative of the tracking-error function (22) yields

$$
\dot{\xi}=H \dot{p}_{e}
$$

with

$$
H=\left[\begin{array}{ccc}
1 & 0 & 0 \\
-\rho\left(\left|x_{e}\right|+\rho\right)^{-2} \operatorname{sgn}\left(x_{e}\right) \tan ^{-1} y_{e} \frac{\rho}{\left(\left|x_{e}\right|+\rho\right)\left(1+y_{e}^{2}\right)} & 1
\end{array}\right] .
$$

Based on (23), we can obtain

$$
\ddot{\xi}=\dot{H} \dot{p}_{e}+H \ddot{p}_{e}
$$

Substituting (21) into (25) yields the tracking-error dynamic equation as follows:

$$
\ddot{\xi}=\dot{H} \dot{p}_{e}+H\left(\dot{F}+\dot{G} z+G M_{0}^{-1}(B \tau-\delta)\right) .
$$

Furthermore, we define a sliding surface $s$ as

$$
s=\dot{\xi}+\alpha \xi+\beta \operatorname{sig}(\xi)^{\gamma}
$$

where $\alpha, \beta>0,1<\gamma<2$, and the notation $\operatorname{sig}(p)^{q}$ as first introduced in [24] is a simplified expression of

$$
\operatorname{sig}(g)^{h}=|g|^{h} \operatorname{sgn}(g) .
$$

The function $\operatorname{sig}(g)^{h}$, for $h>0$ and $\forall g \in R$ is smooth and monotonically increasing and always returns a real number. It has been proved in Appendix B that when the condition $s$ reaches to zero, for any initial $\xi(0)$ and $\dot{\xi}(0)$, the tracking-error function $\xi$ can converge to zero in a finite time $t_{\xi}$ bounded by

$$
t_{\xi} \leq \frac{|\xi(0)|^{\frac{1}{2}}}{\eta_{1}}
$$

where $\eta_{1}=\alpha|\xi|+\beta|\xi|^{\gamma}$. Meanwhile, taking derivative of the sliding surface $s$, one has

$$
\dot{s}=\ddot{\xi}+\alpha \dot{\xi}+\beta \gamma|\xi|^{\gamma-1} \dot{\xi}
$$

Substitute (26) into (30), we obtain

$$
\dot{s}=\dot{H} \dot{p}_{e}+H\left(\dot{F}+\dot{G} z+G M_{0}^{-1}(B \tau-\delta)\right)+\alpha \dot{\xi}+\beta \gamma|\xi|^{\gamma-1} \dot{\xi}
$$


Last, the AFNTSMDC law will be constructed based on the tracking-error function and sliding surface proposed above. Let $\delta=0$ and replace $\tau$ with $\tau_{e q}$. Then, solving (31) for $\dot{s}=0$ leads to

$$
\tau_{e q}=-\left(H G M_{0}^{-1} B\right)^{-1}\left(\dot{H} \dot{p}_{e}+H(\dot{F}+\dot{G} z)+\alpha \dot{\xi}+\beta \gamma|\xi|^{\gamma-1} \dot{\xi}\right) .
$$

Furthermore, a reaching control input $\tau_{r}$ is designed as

$$
\tau_{r}=-\left(H G M_{0}^{-1} B\right)^{-1}\left(K_{1} s+K_{2} \operatorname{sig}(s)^{\mu}+\left(\hat{c_{0}}+\hat{c_{1}}|z|\right) \operatorname{sgn}(s)\right)
$$

where $K_{1}, K_{2}>0,0<\mu<1$; and $\hat{c_{0}}$ and $\hat{c_{1}}$ are updated by the following adaptive law:

$$
\begin{aligned}
& \dot{\hat{c_{0}}}=\zeta_{0}|s| \\
& \dot{\hat{c_{1}}}=\zeta_{1}|z||s|
\end{aligned}
$$

with $\zeta_{0}, \zeta_{1}>0$ to be designed and $\hat{c_{i}}(0) \geq 0(i=0,1)$. Thus, the complete form of the AFNTSMDC law can be obtained and shown in the following lemma.

\subsection{Stability analysis}

The result for the proposed AFNTSMDC law is summarized in the following lemma and stability analysis is provided.

Lemma 1 Consider the NWMR system in (4) and (11), then under the AFNTSMDC law

$$
\tau=\tau_{e q}+\tau_{r}
$$

where $\tau_{e q}$ and $\tau_{r}$ are given in (32) and (33), respectively, the tracking error vector $p_{e}$ converges to zero in a finite time.

Proof Firstly, substituting the control law (36) into (31), one can obtain

$$
\dot{s}=-K_{1} s-K_{2} \operatorname{sig}(s)^{\mu}-\left(\hat{c_{0}}+\hat{c_{1}}|z|\right) \operatorname{sgn}(s)-\left(H G M_{0}^{-1}\right)^{-1} \delta .
$$

Define the adaptive estimation error $\bar{c}_{i}=\hat{c}_{i}-c_{i},(i=0,1)$ and choose the Lyapunov function as

$$
V=\frac{1}{2} s^{2}+\frac{1}{2}\left(\mu_{0} \bar{c}_{0}^{2}+\mu_{1} \bar{c}_{1}^{2}\right)
$$

where $\mu_{0}$ and $\mu_{1}$ are positive numbers. Using (37) and evaluating the derivative of $V$ along this system trajectory with the proposed AFNTSMDC input yields

$$
\begin{aligned}
\dot{V}= & s \dot{s}+\mu_{0} \overline{c_{0}} \dot{\hat{c_{0}}}+\mu_{1} \overline{c_{1}} \dot{\hat{c_{1}}} \\
= & -K_{1} s^{2}-K_{2}|s|^{\mu+1}-\left(\hat{c_{0}}+\hat{c_{1}}|z|\right)|s|-\left(H G M_{0}^{-1}\right)^{-1} \delta|s| \\
& +\mu_{0} \bar{c}_{0} \dot{\hat{c_{0}}}+\mu_{1} \bar{c}_{1} \dot{\hat{c_{1}}} \\
\leq & -\left(\hat{c_{0}}+\hat{c_{1}}|z|\right)|s|-\left|\left(H G M_{0}^{-1}\right)^{-1} \delta\right||s|+\mu_{0} \bar{c}_{0} \zeta_{0}|s| \\
& +\mu_{1} \bar{c}_{1} \zeta_{1}|z||s|
\end{aligned}
$$




$$
\begin{aligned}
= & -\left(\hat{c_{0}}+\hat{c_{1}}|z|\right)|s|-\left|\left(H G M_{0}^{-1}\right)^{-1} \delta\right||s|+\mu_{0} \bar{c}_{0} \zeta_{0}|s| \\
& +\mu_{1} \bar{c}_{1} \zeta_{1}|z||s|-\left(c_{0}+c_{1}|z|\right)|s|+\left(c_{0}+c_{1}|z|\right)|s| \\
= & -\left(c_{0}+c_{1}|z|-\left|\left(H G M_{0}^{-1}\right)^{-1} \delta\right|\right)|s|+\left(\mu_{0} \zeta_{0}-1\right) \bar{c}_{0}|s| \\
& +\left(\mu_{1} \zeta_{1}-1\right)|z| \bar{c}_{1}|s| \\
\leq & \left(c_{0}+c_{1}|z|-\left|\left(H G M_{0}^{-1}\right)^{-1} \delta\right|\right)|s|+\left(\mu_{0} \zeta_{0}-1\right)\left|\bar{c}_{0}\right||s| \\
& +\left(\mu_{1} \zeta_{1}-1\right)|z|\left|\bar{c}_{1}\right||s| .
\end{aligned}
$$

To make the expression compact, we define the following symbols as:

$$
\begin{aligned}
\epsilon_{s} & =c_{0}+c_{1}|z|-\left|\left(H G M_{0}^{-1}\right)^{-1} \delta\right| \\
\epsilon_{0} & =\left(\mu_{0} \zeta_{0}-1\right)|s| \\
\epsilon_{1} & =\left(\mu_{1} \zeta_{1}-1\right)|z||s| .
\end{aligned}
$$

Then, if $\epsilon_{0}, \epsilon_{1}$, and $\epsilon_{2}>0,(39)$ can be rewritten as

$$
\begin{aligned}
\dot{V} & \leq-\epsilon_{s} \sqrt{2} \frac{|s|}{\sqrt{2}}-\epsilon_{0} \sqrt{2 \mu_{0}^{-1}} \sqrt{\frac{\mu_{0}}{2}}\left|\bar{c}_{0}\right|-\epsilon_{1} \sqrt{2 \mu_{1}^{-1}} \sqrt{\frac{\mu_{1}}{2}}\left|\bar{c}_{1}\right| \\
& \leq \eta_{2}\left(\frac{|s|}{\sqrt{2}}+\sqrt{\frac{\mu_{0}}{2}}\left|\overline{c_{0}}\right|+\sqrt{\frac{\mu_{1}}{2}}\left|\overline{c_{1}}\right|\right) \\
& \leq \eta_{2} V^{\frac{1}{2}}
\end{aligned}
$$

where

$$
\eta_{2}=\min \left\{\sigma_{s} \sqrt{2}, \sigma_{0} \sqrt{2 \mu_{0}^{-1}}, \sigma_{1} \sqrt{2 \mu_{1}^{-1}}\right\} .
$$

It is obvious that $\sigma_{s}>0$, and for any $\zeta_{1}$ and $\zeta_{2}$, there always exits positive constants $\mu_{0}$ and $\mu_{1}$ such as $\mu_{0} \zeta_{0}>0$ and $\mu_{1} \zeta_{1}>0$, which imply $\eta_{2}>0$. Therefore, according to the result in Appendix A, the inequality (40) satisfies the finite-time stability criterion. More specific, $V$ will converge from any initial condition $V(0)$ to zero in a finite time $t_{s}$ shown in the following equation:

$$
t_{s} \leq \frac{2 V^{\frac{1}{2}}(0)}{\eta_{2}}
$$

This implies that the sliding variable $s$ and the estimation error $\overline{c_{i}}$ will converge to zero in a finite time $t_{s}$.

When sliding surface is first arrived (i.e., $s=0$ and $\dot{\xi}=-\alpha \xi-\beta \operatorname{sig}(\xi)^{\gamma}$ ), the finite-time converging condition of $\xi$ is met. After a finite time $t_{\xi}$ when $\xi=0$, according to the definition of $\xi$ in (22), we have

$$
\left\{\begin{array}{l}
x_{e}=0 \\
\phi_{e}=-\tan ^{-1} y_{e} .
\end{array}\right.
$$

Substituting the condition (43) into (18), the dynamics of $y_{e}$ can be written as

$$
\begin{aligned}
\dot{y_{e}} & =-v_{r} \sin \left(\tan ^{-1} y_{e}\right) \\
& =-\frac{v_{r} y_{e}}{\sqrt{1+y_{e}^{2}}} .
\end{aligned}
$$

To investigate the stability of the dynamics of $y_{e}$, we choose a Lyapunov candidate $V_{y e}=\frac{1}{2} y_{e}^{2}$. Thus, $\dot{V}_{y e}$ can be expressed as

$$
\dot{V}_{y e}=-\frac{v_{r} y_{e}^{2}}{\sqrt{1+y_{e}^{2}}}
$$




$$
\begin{aligned}
& \leq-\frac{v_{r}\left|y_{e}\right|}{\sqrt{1+y_{e}^{2}}} \sqrt{2} \frac{\left|y_{e}\right|}{\sqrt{2}} \\
& =-\sqrt{2} \eta_{3} V_{y e}^{\frac{1}{2}}
\end{aligned}
$$

where $\eta_{3}=\frac{v_{r}\left|y_{e}\right|}{\sqrt{1+y_{e}^{2}}}$ and $v_{r}>0$. According to Appendix A, $y_{e}$ converges to zero in the finite time satisfying

$$
t_{y e} \leq-\frac{\sqrt{2} V^{\frac{1}{2}}(0)}{\eta_{3}}
$$

Recall the condition in (43). When $y_{e}$ reaches zero, $\phi_{e}$ reaches zero at the same time. Therefore, under the proposed AFNTSMDC law, the x-axis tracking error $x_{e}$ converges from any initial condition to zero in a finite time of $t_{x e}=t_{s}+t_{\xi}$ and it takes additional finite time $t_{y e}$ for $y_{e}$ and $\phi_{e}$ to reach zero. The proof is thus completed.

Remark 1 The controller (36) requires the inverse of the matrix $H G M_{0}^{-1} B$. Similar issue has appeared in $[13,15]$ while examining the singularity of the control laws. In our method, the singularity issue can be resolved by the proposed tracking-error function (22). This can be straightly justified by the determinant of the matrix $H G M_{0}^{-1} B$ as follows:

$$
\operatorname{det}\left|H G M_{0}^{-1} B\right|=-\frac{2 b}{r m_{0} I_{0}}\left(\frac{\rho}{\left(\left|x_{e}\right|+\rho\right)\left(1+y_{e}^{2}\right)} x_{e}+1\right) .
$$

It can be seen that for $0<\rho<1$, the function $\frac{\rho}{\left(\left|x_{e}\right|+\rho\right)\left(1+y_{e}^{2}\right)} x_{e} \neq-1$ because of the functions $\frac{\rho x_{e}}{\left|x_{e}\right|+\rho} \in(-1,1)$ and $\frac{1}{1+y_{e}^{2}} \in(0,1]$. Therefore, the inverse of $H G M_{0}^{-1} B$ is finite.

Remark 2 In the adaptation law (34)-(35), the sliding variable $s$ is generally chattering around zero due to system uncertainties and measurement noises, resulting in overly large estimations of $c_{i}$ causing control input saturation. Hence, the dead zone technique [25] can be employed in practice to moderate this issue. More specifically, the following rules are implemented:

$$
\begin{aligned}
& \begin{cases}\dot{\hat{c_{0}}}=\zeta_{0}|s|, & \text { for }|s|>\varepsilon \\
\dot{\hat{c_{0}}}=0, & \text { for }|s| \leq \varepsilon\end{cases} \\
& \begin{cases}\dot{\hat{c}_{1}}=\zeta_{1}|z||s|, & \text { for }|s|>\varepsilon \\
\dot{\hat{c_{1}}}=0, & \text { for }|s| \leq \varepsilon\end{cases}
\end{aligned}
$$

where $\varepsilon>0$ is a small threshold value selected as 0.05 in our case. It is clear that when $s$ is within the region $\varepsilon, \hat{c_{0}}$ and $\hat{c_{1}}$ will not increase but retain the present values. One can easily verify that then $|s|<\varepsilon$, (40) still holds. Therefore, the finite-time stability property is still retained in practice.

Remark 3 The boundary layer technique can be used to compromise between control accuracy and the chattering induced by the reaching control. This can be accomplished by replacing the signum function in the reaching law (33) with a saturation function given by

$$
\operatorname{sat}(s)= \begin{cases}\operatorname{sgn}(s), & \text { for }|s|>\varrho \\ s \varrho^{-1}, & \text { for }|s| \leq \varrho\end{cases}
$$


where $\varrho$ denotes the boundary layer thickness and $\varrho=\left[\begin{array}{cc}0.04 & 0 \\ 0 & 0.04\end{array}\right]$ is chosen in our case to reach a balance between chattering reduction and acceptable tracking errors.

\subsection{Control parameters selection}

During the implementation, trade-offs between the desired tracking performance and other factors such as control input saturation, control command smoothness, and measurement noises are expected. In the following, we will discuss the controller parameters selection guideline for the proposed AFNTSMDC law and give their values for the NWMR under study.

1) Selection of $\alpha, \beta, \gamma$ : The parameters $\alpha, \beta$, and $\gamma$ affect the dynamic behaviors of the sliding surface $s$ in (27). An increment of these parameters can lead to faster convergence of $\xi$ towards zero but request an increased control input in (36). For the NWMR constructed for the experiment, $\alpha=\left[\begin{array}{ll}7 & 0 \\ 0 & 7\end{array}\right], \beta=\left[\begin{array}{ll}7 & 0 \\ 0 & 6\end{array}\right]$, and $\gamma=1.67$ are chosen.

2) Selection of $\rho$ : Before $x_{e}$ reaches zero, a smaller value of $\rho$ in the trackingerror function (22) can increase the convergence rate of $y_{e}$ and $\phi_{e}$. However, it may invoke oscillations in transient response of $\phi_{e}$. Thus, $\rho$ is set to be 0.8 .

3) Selection of $K_{1}, K_{2}, \mu$ : The control parameters $K_{1}, K_{2}$, and $\mu$ in the reaching law (33) dominate the system robustness. Increasing the values of $K_{1}$ and $K_{2}$ strengthens the system robustness at the cost of control signal smoothness. Meanwhile, $\mu$ can balance the control signal chattering and the robustness. In our case, $K_{1}=\left[\begin{array}{ll}9 & 0 \\ 0 & 4\end{array}\right], K_{2}=\left[\begin{array}{cc}15 & 0 \\ 0 & 5\end{array}\right]$, and $\mu=0.8$ are chosen in the implementation.

4) Selection of $c_{0}(0), c_{1}(0), \zeta_{0}, \zeta_{1}: c_{0}(0), c_{1}(0)$ denote the initial values of $c_{0}$, $c_{1}$. A good guess of them will reduce the adaptation time. The adaptive gains $\zeta_{0}, \zeta_{1}$ in $(34)-(35)$ determine the convergence rate of the adaptive estimation error. However, a large value of them may cause control input saturation and overshoots. Thus, we find $c_{0}(0)=\left[\begin{array}{cc}14 & 0 \\ 0 & 8\end{array}\right], c_{1}(0)=\left[\begin{array}{cc}14 & 0 \\ 0 & 6\end{array}\right]$, $\zeta_{0}=\left[\begin{array}{cc}0.3 & 0 \\ 0 & 0.4\end{array}\right], \zeta_{1}=\left[\begin{array}{cc}0.4 & 0 \\ 0 & 0.5\end{array}\right]$ are sufficient for the experiments.

\section{Experimental results}

To demonstrate the effectiveness of the proposed AFNTSMDC method in the presence of external disturbances and load variations, experiments are conducted on the NWMR shown in Fig. 4. In addition, experimental comparisons are made with other existing control methods, i.e., a classic kinematic control (CKC) method [19], a recently proposed robust sliding mode kinematic 


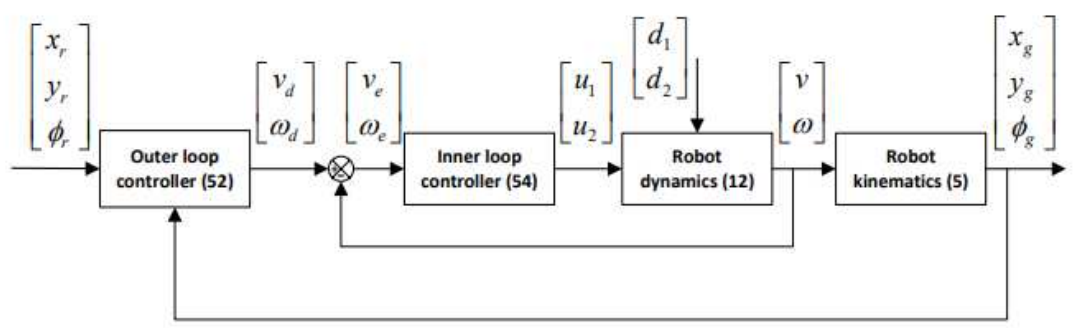

Fig. 3: Block diagram of an inner-outer control structure for the NWMR system [12].

control (RSMKC) method [15], and a conventional sliding mode dynamic control (CSMDC) method. In the experiments, the feedback signals in Fig. 3 are calculated based on the available motor encoder signals.

\subsection{Control methods for comparison}

Classic Kinematic Control ( $C K C$ ): An inner-outer loop control design scheme is a classic control scheme. As shown in Fig. 3, the inner velocity controller is designed based on the dynamic model. Then, the outer loop controller design is based on the kinematic model. An outer loop CKC law is given in the following form[19]:

$$
z_{d}=K_{E} p_{e}+K_{D}
$$

with

$$
z_{d}=\left[\begin{array}{c}
v_{d} \\
\omega_{d}
\end{array}\right], K_{E}=\left[\begin{array}{ccc}
k_{e 1} & 0 & 0 \\
0 & v_{r} k_{e 2} & 0
\end{array}\right], K_{D}=\left[\begin{array}{c}
v_{r} \cos \phi_{e} \\
v_{r} k_{e 3} \sin \phi_{e}+\omega_{r}
\end{array}\right]
$$

where $k_{e i}(i=1,2,3)$ are all positive constants which are set to be $1.7,1.7$, and 1.5 in this experiment.

Robust Sliding Mode Kinematic Control (RSMKC): Similarly, an RSMKC method is proposed in [15]

$$
\left\{\begin{array}{l}
s_{r}=\left[\begin{array}{c}
k_{r 1} \phi_{e}+v_{r} \tan ^{-1} y_{e} \\
x_{e}
\end{array}\right] \\
z_{d}=-B_{r}^{-1}\left(A_{r} F_{r}+D_{r}+K_{r} \operatorname{sgn}\left(s_{r}\right)\right)
\end{array}\right.
$$

where

$$
\begin{aligned}
& A_{r}=\left[\begin{array}{ccc}
0 & \frac{v_{r}}{1+y_{e}^{2}} & k_{r 1} \\
1 & 0 & 0
\end{array}\right], B_{r}=\left[\begin{array}{cc}
0 & -\left(k_{r 1}+\frac{v_{r} x_{e}}{1+y_{e}^{2}}\right) \\
-1 & y_{e}
\end{array}\right] \\
& F_{r}=\left[\begin{array}{c}
v_{r} \cos \phi_{e} \\
v_{r} \sin \phi_{e} \\
\omega_{r}
\end{array}\right], D_{r}=\left[\begin{array}{c}
v_{r} \tan ^{-1} y_{e} \\
0
\end{array}\right], K_{r}=\left[\begin{array}{cc}
k_{r 2} & 0 \\
0 & k_{r 3}
\end{array}\right]
\end{aligned}
$$


Table 1: Model parameters of the NWMR

\begin{tabular}{lll}
\hline Parameter & Symbol & $\begin{array}{l}\text { Nominal } \\
\text { value }\end{array}$ \\
\hline Mass of the NWMR & $m_{0}$ & $4.500 \mathrm{~kg}$ \\
Moment of inertia of the NWMR & $I_{0}$ & $0.560 \mathrm{kgm}^{2}$ \\
Wheel radius & $r$ & $0.042 \mathrm{~m}$ \\
Distance from mass center to wheel & $b$ & $0.186 \mathrm{~m}$ \\
\hline
\end{tabular}

where $k_{r i}(i=1,2,3)$ are tuning parameters which are set to $0.40,0.02$, and 0.02 , respectively.

It is worth noting that both kinematic controllers presented above are coupled with an inner velocity controller which is designed for the actual velocity to follow the desired velocity generated by the kinematic controller. A PI velocity controller is used in experiments and it can be described as

$$
z=k_{P} z_{e}(T)+k_{I} \int_{0}^{T} z_{e}(t) d t
$$

where $z_{e}=z_{d}-z$ which is the velocity tracking error, $k_{P}$ and $k_{I}$ are tuning parameters and they are set to 30 and 250 in our case, respectively.

Conventional Sliding Mode Dynamic Control (CSMDC): For comparison, a CSMDC method is listed below whose control scheme is the same as the one discussed in Section II. However, the sliding surface and reaching law are designed based on the conventional SMC method which is given as

$$
\left\{\begin{array}{l}
s_{2}=\lambda_{c} \xi+\dot{\xi} \\
\tau_{e q 2}=-\left(H G M_{0}^{-1} B\right)^{-1}\left(\lambda_{c} \dot{\xi}+\dot{H} \dot{p}_{e}+H(\dot{F}+\dot{G} V)\right) \\
\tau_{r 2}=-\left(H G M_{0}^{-1} B\right)^{-1} K_{c} \operatorname{sgn}\left(s_{2}\right) \\
\tau_{2}=\tau_{e q 2}+\tau_{r 2}
\end{array}\right.
$$

with

$$
K_{c}=\left[\begin{array}{cc}
k_{c 1} & 0 \\
0 & k_{c 2}
\end{array}\right], \lambda_{C}=\left[\begin{array}{cc}
\lambda_{c 1} & 0 \\
0 & \lambda_{c 2}
\end{array}\right]
$$

where $\xi$ is with $(22) ; k_{c i}$ and $\lambda_{c i}(i=1,2)$ are tuning parameters which are chosen as $21,16,3$ and 4 , respectively.

\subsection{Experimental platform}

Our experimental setup for the NWMR is shown in Fig. 4 with its nominal physical parameters listed in Table 1. It consists of two dc-motors (Maxon) which are integrated with encoders to measure the rotary angles. Two motor drivers (Maxon ESCON-36/2) are also used to control the motor power. Two caster wheels are located at the rear of the robot. The data acquisition and 


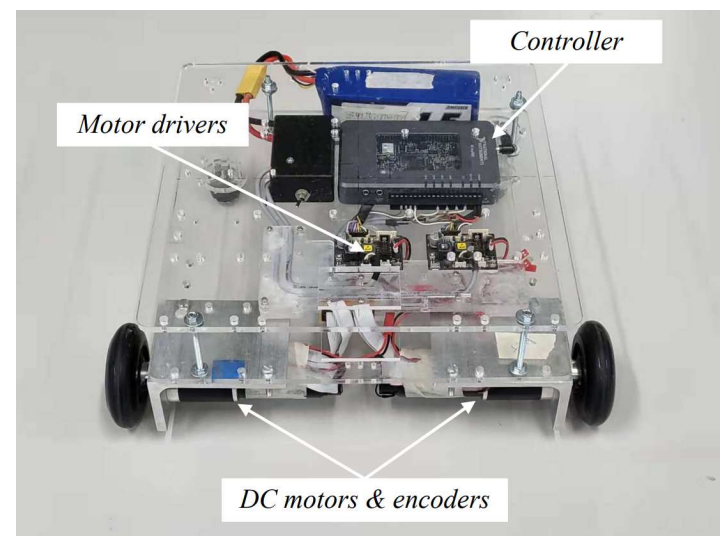

Fig. 4: Experimental platform of a nonholonomic wheeled mobile robot.

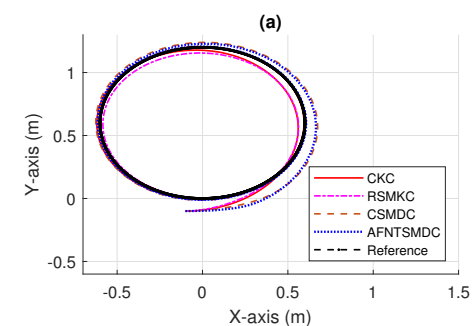

(b)

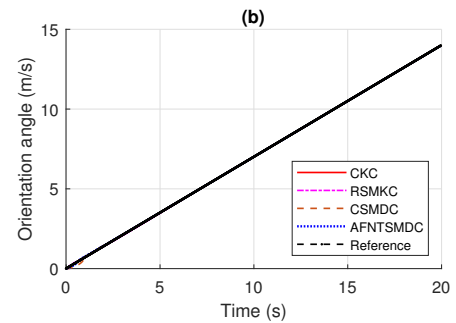

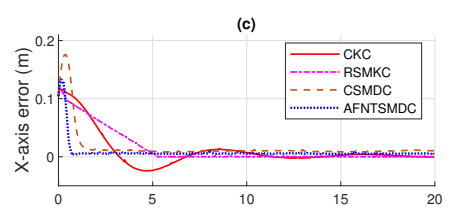

(d)

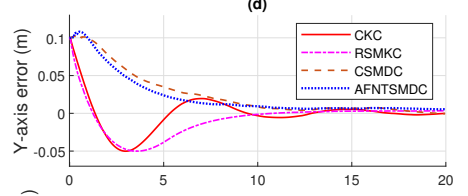

(e)

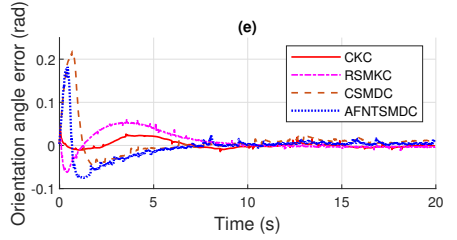

Fig. 5: Circular tracking with initial posture offset. (a) Trajectory profiles; (b) Orientation angles; (c) Tracking errors of X-axis displacement; (d) Tracking errors of Y-axis displacement; (e) Tracking errors of orientation angle.

controller are implemented with the real-time microcontroller (NI myRIO). The sampling period is set to 0.01 seconds for all controllers implemented. The feedback posture signals of the NWMR are obtained by using a posture estimator with the motor encoder signals as the system input.

\subsection{Circular path tracking performance with initial posture offset}

A circular path is typically used for testing the track-following performance by mobile robots. In the experiments conducted, the desired circular path is 
(a)

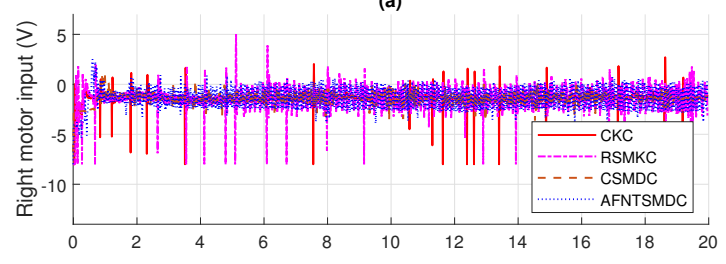

(b)

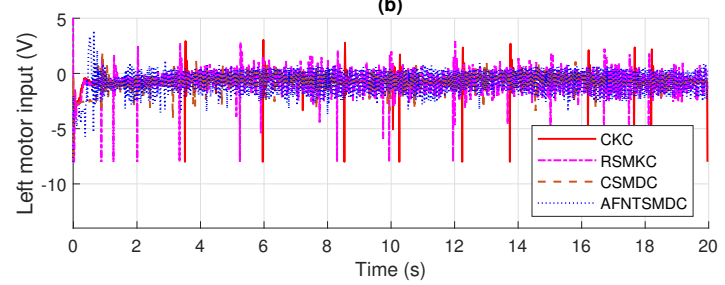

Fig. 6: Control input with initial posture offset. (a) Right motor voltage input; (b) Left motor voltage input.

configured with an angular speed of $0.70 \mathrm{rads} / \mathrm{s}$ and a radius of $0.60 \mathrm{~m}$. In addition, the initial posture offset is set to be $(-0.10,-0.10,0)$.

Experimental results on the trajectory and tracking error profiles are shown in Fig. 5. We can see that the AFNTSMDC method achieves the fastest convergence rate and the least oscillations. In particular, Fig. 5(c) shows that the convergence of $\mathrm{X}$-axis tracking error under AFNTSMDC is with a settling time of $0.68 \mathrm{~s}$ which is $88.95 \%, 85.71 \%$, and $83.36 \%$ faster than that of the CKC, RSMKC, and CSMDC, respectively. Moreover, Fig. 5(d)-(e) show that both $\mathrm{Y}$-axis and orientation tracking error converge to zero as desired. Fig. 6 presents the control inputs of all controllers, where the AFNTSMDC exhibits persistently smooth control signals without singularity issue that matches the theoretical design. Nevertheless, due to the impact of measurement noise, there is still a small level of chattering in the control input, which is inevitable in the experiments but has not caused any implementation issue in our case.

\subsection{Circular path tracking performance with load uncertainty}

To further verify the performance robustness in the presence of modelling uncertainties, we place a $3.60-\mathrm{kg}$ cylindrical payload on the mass center of the mobile robot, i.e., making $\Delta M=\left[\begin{array}{cc}3.60 & 0 \\ 0 & 0.01\end{array}\right]$. Meanwhile, the circular path reference is still used on the control system with an initial posture offset set on the robot. Fig. 7 shows that the AFNTSMDC maintains its advantages with the shortest settling time in the tracking error because the payload uncertainties have been explicitly considered during the design process as shown in (11). Whereas the kinematic controllers (i.e., CKC and RSMKC) suffer from severe performance degradation due to their weak robustness against system 


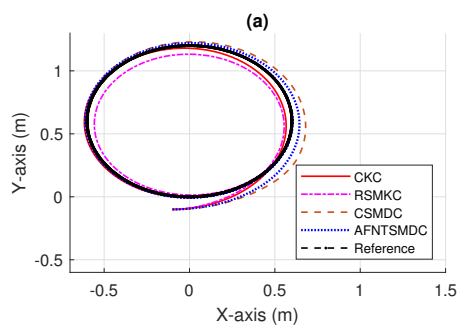

(b)

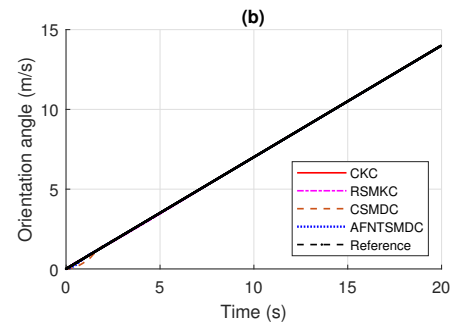

(c)

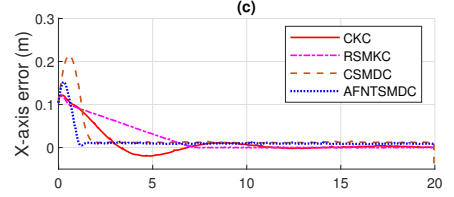

(d)
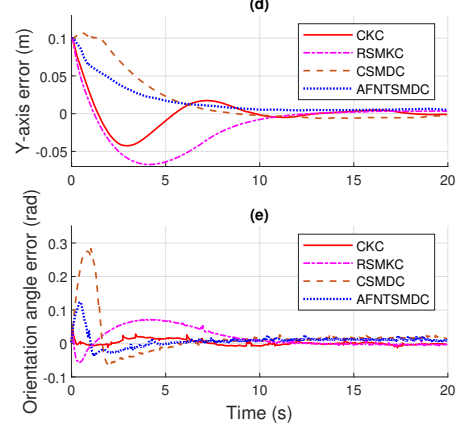

Fig. 7: Circular tracking with the load. (a) Trajectory profiles; (b) Orientation angles; (c) Tracking errors of X-axis displacement; (d) Tracking errors of Y-axis displacement; (e) Tracking errors of orientation angle.

(a)

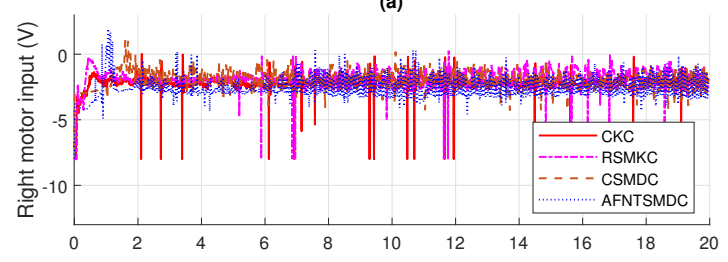

(b)

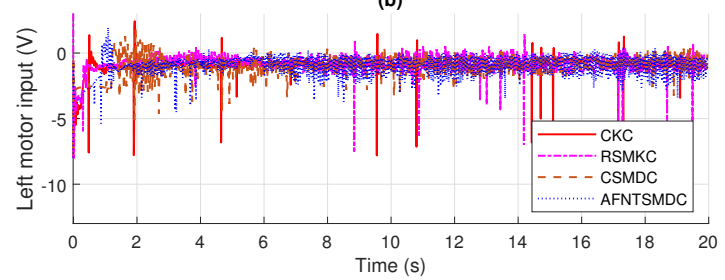

Fig. 8: Control input with the load. (a) Right motor voltage input; (b) Left motor voltage input.

uncertainties. From the control input in Fig. 8, it can be observed that there is a significant increment in the amplitude and the number of spikes in control input signals except those under CSMDC and AFNTSMDC. The increased amplitudes in Fig. 8(b) are 39.54\%, 40.83\%, 35.41\%, and $24.05 \%$ for the CKC, RSMKC, CSMDC, and AFNTSMDC, respectively. Hence, this experiment validates that the proposed controller is more robust against modelling uncertainties in comparison with other control methods. 
(a)

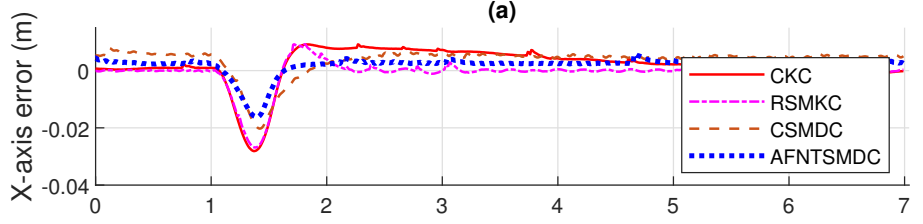

(b)

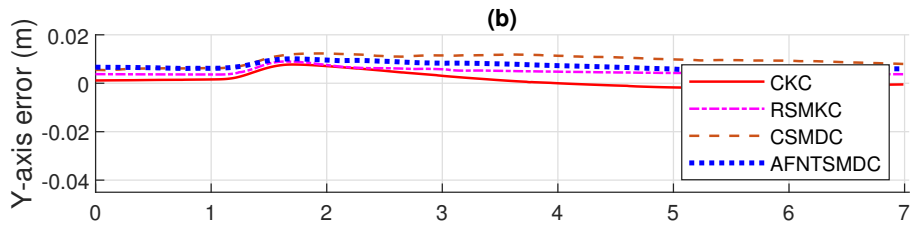

(c)

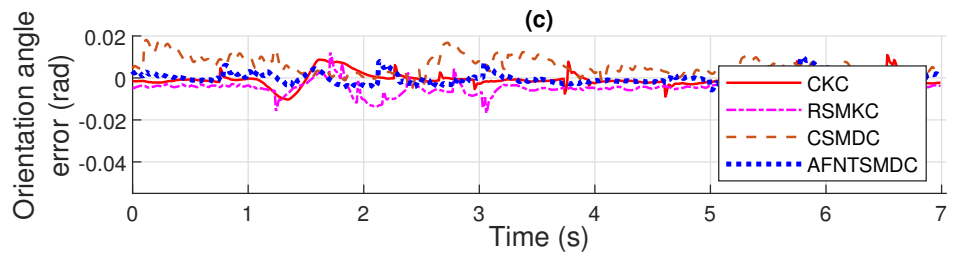

Fig. 9: Circular tracking under external disturbances. (a) Tracking errors of $\mathrm{X}$-axis displacement; (b) Tracking errors of Y-axis displacement; (c) Tracking errors of orientation angle.

(a)

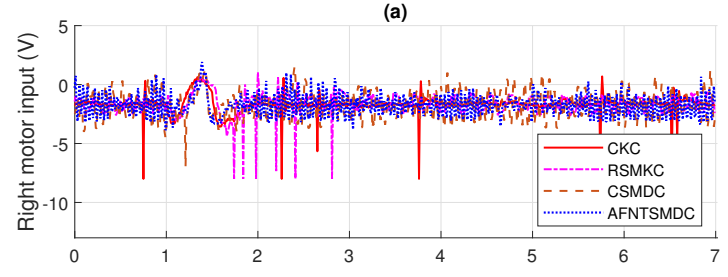

(b)

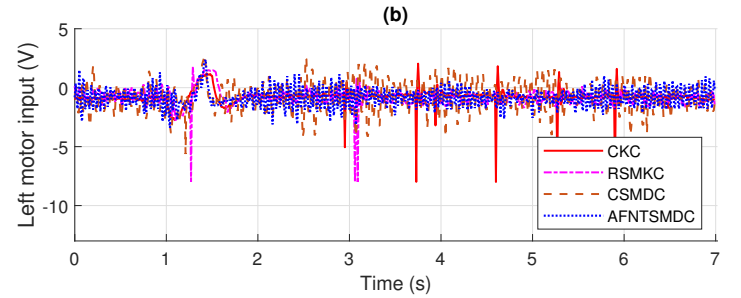

Fig. 10: Control input under external disturbances. (a) Right motor voltage input; (b) Left motor voltage input.

\subsection{Circular path tracking performance under external disturbances}

The disturbances $d_{1}$ and $d_{2}$ as shown in Fig. 2 and Fig. 3 are set as shock disturbances acting on the wheels of the NWMR. The disturbance behaves as 
a half-sine waveform with a duration of $0.5 \mathrm{~s}$ and an amplitude of $6.0 \mathrm{~V}$. The disturbance can be modelled as

$$
\left\{\begin{array}{lrl}
d_{1}=d_{2}=6|\sin (2 \pi t)| & 12 \mathrm{~s} \leq t \leq 12.5 \mathrm{~s} \\
d_{1}=d_{2}=0 & \text { Other time. }
\end{array}\right.
$$

One of the most notable features of the AFNTSMDC is the fast convergence rate in response to an external disturbance. It can be seen from Fig. 9(a) that the tracking error under the AFNTSMDC has a peak value of $16.2 \mathrm{~mm}$, which is $42.32 \%, 39.59 \%$, and $19.93 \%$ smaller than the peaks values under the CKC, RSMKC, and CSMDC. In addition, the settling time of X-axis tracking error is $0.27 \mathrm{~s}$ under AFNTSMDC in comparison with $2.23 \mathrm{~s}, 0.62 \mathrm{~s}$, and $0.55 \mathrm{~s}$ under the CKC, RSMKC, and CSMDC, respectively. While obtaining stronger robustness, only the same level of control inputs is required under AFNTSMDC, which can be seen from Fig. 10. Thus, the results verify that the AFNTSMDC is more robust and faster in terms of disturbance rejection capability and settling-time convergence.

In summary, the proposed AFNTSMDC controller performs the most robustly and fastest in the trajectory tracking control for the NWMR. It is also worth noting that the tracking errors under all controllers still contain a small amount of steady-state error in practice due to the coarse sensor resolution and the trade-off for reducing the control chattering, which will be improved in our future work.

\section{Conclusion}

In this paper, we developed an AFNTSMDC method for the NWMR system to accomplish trajectory tracking control in a finite time. This proposed control scheme simplifies the control structure and unifies the design process in comparison with the classic inner-outer loop control scheme. Accordingly, the control parameter tuning is moderated since there is no need to design two individual controllers for different objectives. Furthermore, the AFNTSMDC method resolves the singularity issue and possesses strong robustness against external disturbances and modelling uncertainties. To verify the effectiveness of the controller on the NWMR system, a series of circular trajectory tracking experiments have been conducted. The results demonstrate the effectiveness of the AFNTSMDC method with stronger robustness and faster convergence rate when compensating for initial posture offset, load uncertainty, and external disturbances compared to the existing CKC, RSMKC, and CSMDC methods.

In our future work, we will investigate the chattering-free reaching law to inherently eliminate the chattering phenomenon without sacrificing tracking accuracy. 


\section{Declarations}

Conflict of interest/Competing interests. The authors declare that they have no conflict of interest.

Availability of data and materials. The data collected in this study is available from the corresponding author on reasonable request.

\section{Appendix A}

Given the following first-order nonlinear differential inequality:

$$
\dot{V}(x)+j V^{f}(x) \leq 0
$$

where $V(x)$ represents a positive Lyapunov function with respect to the state $x \in R, j>0,0<f<1$, then for any given initial condition $V(x(0))=V(0)$, the function $V(x)$ converges to the origin in the finite time given by

$$
t_{v} \leq \frac{V^{1-f}(0)}{j(1-f)}
$$

The derivation is referred to $[26,27]$ and references therein.

\section{Appendix B}

For the system $\dot{\xi}=-\alpha \xi-\beta \operatorname{sig}(\xi)^{\mu}$, define the Lyapunov function $V=\frac{1}{2} p_{e}^{2}$

$$
\begin{aligned}
\dot{V} & =\xi \dot{\xi} \\
& =-\alpha \xi^{2}-\beta|\xi|^{\mu+1} \\
& \leq-\left(\alpha|\xi|-\beta|\xi|^{\mu}\right) \sqrt{2} \frac{|\xi|}{\sqrt{2}} \\
& =-\sqrt{2} \eta_{1} V^{\frac{1}{2}}(t)
\end{aligned}
$$

where $\eta_{1}=\alpha|\xi|-\beta|\xi|^{\mu}$. According to Appendix A, e converges to zero in the finite time satisfying

$$
t_{\xi} \leq \frac{\sqrt{2} V^{\frac{1}{2}}(0)}{\eta_{1}}
$$

\section{References}

[1] Fabregas, E., Farias, G., Aranda-Escolástico, E., Garcia, G., Chaos D., Dormido-Canto, S., Bencomo, S.D.: Simulation and Experimental Results of a New Control Strategy For Point Stabilization of Nonholonomic Mobile Robots. IEEE Trans. Ind. Electron. 67(8),6679-6687 (2019). 
[2] Brockett, R.W.: Asymptotic stability and feedback stabilization. Differ. Geometric Control Theory 27(1),181-191 (1983).

[3] Fierro, R., Lewis, F.L.: Control of a nonholonomic mobile robot: Backstepping kinematics into dynamics. J. Robot. Syst. 14(3),149-163 (1997).

[4] Fukao, T., Nakagawa, H., Adachi, N.: Adaptive tracking control of a nonholonomic mobile robot. IEEE Trans. Robot. Autom. 16(5),609-615 (2000).

[5] Fierro, R., Lewis, F.L.: Control of a nonholonomic mobile robot using neural networks. IEEE Trans. Neural Netw. 9(4),589-600 (1998).

[6] Zeng-Guang, H., An-Min, Z., Long, C., Min, T.: Adaptive Control of an Electrically Driven Nonholonomic Mobile Robot via Backstepping and Fuzzy Approach. IEEE Trans. Control Syst. Technol. 17(4),803-815 (2009).

[7] Huang, J., Wen, C., Wang, W., Jiang, Z.P.: Adaptive output feedback tracking control of a nonholonomic mobile robot. Automatica 50(3),821831 (2014).

[8] Zheng, J., Wang, H., Man, Z., Jin, J., Fu, M.: Robust Motion Control of a Linear Motor Positioner Using Fast Nonsingular Terminal Sliding Mode. IEEE ASME Trans. Mechatron. 20(4),1743-1752 (2015).

[9] Sun, Z., Xie H., Zheng, J., Man, Z., He, D.: Path-following control of Mecanum-wheels omnidirectional mobile robots using nonsingular terminal sliding mode. Mech. Syst. Signal Process. 147,107128 (2021).

[10] Yang, J.M., Kim, J.H.: Sliding mode control for trajectory tracking of nonholonomic wheeled mobile robots. IEEE Trans. Robot. 15(3),578-587 (1999).

[11] Chwa, D.: Sliding-mode tracking control of nonholonomic wheeled mobile robots in polar coordinates. IEEE Trans. Control Syst. Technol. 12(4),637644 (2004).

[12] Xie, H., Zheng, J., Chai, R., Nguyen, H.T.: Robust tracking control of a differential drive wheeled mobile robot using fast nonsingular terminal sliding mode. Comput. Electr. Eng. 96,107488 (2021).

[13] Youssef, E., Martins, N.A., Pieri, D., Moreno U.F.: PD-Super-Twisting Second Order Sliding Mode Tracking Control for a Nonholonomic Wheeled Mobile Robot. In: Proceedings of the 19th World Congress the International Federation of Automatic Control, Cape Town, South Africa. August 24-29, pp.3827-3832, (2014). 
[14] Begnini, M., Bertol, D.W., Martins, N.A.: A robust adaptive fuzzy variable structure tracking control for the wheeled mobile robot: Simulation and experimental results. Control Eng. Pract. 64,27-43 (2017).

[15] Nath, K., Yesmin, A., Nanda, A., Bera, M.K.: Event-Triggered SlidingMode Control of Two Wheeled Mobile Robot: An Experimental Validation. IEEE Trans. Emerg. Sel. Topics Power Electron. 2(3),218-226 (2021).

[16] Mobayen, S.: Finite-time tracking control of chained-form nonholonomic systems with external disturbances based on recursive terminal sliding mode method. Nonlinear Dyn. 80(1-2),669-683 (2015).

[17] Cui, M., Liu, W., Liu, H., Jiang, H., Wang, Z.: Extended state observerbased adaptive sliding mode control of differential-driving mobile robot with uncertainties. Nonlinear Dyn. 83(1-2),667-683 (2016).

[18] Ou, M., Sun, H., Zhang, Z., Gu, S.: Fixed-time trajectory tracking control for nonholonomic mobile robot based on visual servoing. Nonlinear Dyn. (2022).

[19] Kanayama, Y., Kimura, Y., Miyazaki, F., Noguchi, T.: A stable tracking control method for an autonomous mobile robot. In: Proceedings of the IEEE international conference on robotics and automation, Cincinnati, OH, USA, pp. 384- 389 (1990).

[20] Zhang, Y., Liu, G., Luo, B.: Finite-time cascaded tracking control approach for mobile robots. Inf. Sci. 284,31-43 (2014).

[21] Shi, S., Yu, X., Khoo, S.: Robust finite-time tracking control of nonholonomic mobile robots without velocity measurements. Int. J. Control $\mathbf{8 9}(2), 411-423(2016)$.

[22] Zhai, J.Y., Song, Z.B.: Adaptive sliding mode trajectory tracking control for wheeled mobile robots. Int. J. Control 92(10),2255-2262 (2019).

[23] Shao, K., Zheng, J., Huang, K., Wang, H., Man, Z., Fu, M.: Finite-Time Control of a Linear Motor Positioner Using Adaptive Recursive Terminal Sliding Mode. IEEE Trans. Ind. Electron. 67(8),6659-6668 (2020).

[24] Haimo, V.T.: Finite time controllers. SIAM J. Control Optim. 24(4),760770 (1986).

[25] Utkin, V.I.: Sliding modes in control and optimization, Springer Science Business Media, (2013).

[26] Moulay, E., Perruquetti, W.: Finite time stability and stabilization of a class of continuous systems. J. Math. Anal. 323(2),1430-1443 (2006). 
[27] Plestan, F., Shtessel, Y., Brégeault, V., Poznyak, A.: New methodologies for adaptive sliding mode control. Int. J. Control 83(9),1907-1919 (2010). 